\title{
Experiência Inicial de um Programa de TAVI: Análise da Decisão Anestésica e sua Evolução
}

\author{
Initial Experience of a TAVI Program: Analysis of the \\ Anesthetic Decision and its Evolution
}

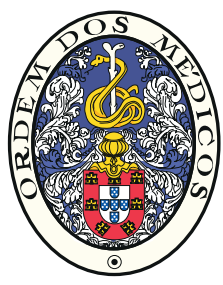

\author{
Ana MARTINS $\square^{1}$, Maria de Lurdes CASTRO ${ }^{1}$, Isabel FRAGATA ${ }^{1}$ \\ Acta Med Port 2019 Feb;32(2):126-132 - https://doi.org/10.20344/amp.10982
}

\begin{abstract}
RESUMO
Introdução: A implantação percutânea de válvula aórtica constitui uma opção menos invasiva de substituição valvular. O número de procedimentos sob anestesia local com sedação tem vindo a crescer com o aumento da experiência da equipa e os acessos cada vez menos invasivos. O trabalho tem como objetivo a descrição da evolução da técnica anestésica utilizada nos doentes submetidos a implantação percutânea de válvula aórtica no nosso centro ao longo dos anos, e sua comparação.

Material e Métodos: Estudo retrospetivo em 149 doentes consecutivos submetidos a implantação percutânea de válvula aórtica no Hospital de Santa Marta (janeiro de 2010 a dezembro de 2016). Os dados foram colhidos a partir dos registos peri-procedimento e estratificados de acordo com a técnica anestésica.

Resultados: Da amostra recolhida, $57,0 \%$ dos doentes eram do sexo feminino, com mediana idade 82 [58 - 95] anos. A maioria dos doentes foi submetida a anestesia geral (68,5\%). Verificou-se menor duração do procedimento (120 [60 - 285] vs 155 [30 - 360]) e menor número de doentes com necessidade de administração de vasopressores na implantação percutânea de válvula aórtica (61,8\% vs $28,3 \%)-p<0,05$. Não se registaram diferenças referentes à duração do internamento (9 [4 - 59] vs 10 [3 - 87]), complicações periprocedimento $(66,0 \%$ vs $72,5 \%)$, reinternamento $(4,3 \%$ vs $3,9 \%)$, mortalidade aos 30 dias $(2,1 \%$ vs $4,9 \%)$ e 1 ano $(6,4 \%$ vs $7,8 \%)$ $-p>0,05$. O número de implantações percutâneas de válvula aórtica realizados sob anestesia local com sedação aumentou ao longo dos anos.
\end{abstract}

Discussão: A escolha da técnica anestésica tende a variar consoante as características do doente, a experiência e preferência da equipa.

Conclusão: Os resultados da anestesia local com sedação são similares aos da anestesia geral, tendo o aumento do número de procedimentos de implantação percutânea de válvula aórtica sob anestesia local com sedação acompanhado a tendência de menor invasibilidade do procedimento.

Palavras-chave: Anestesia Geral; Anestesia Local; Implante de Prótese de Válvula Cardíaca; Substituição Percutânea da Válvula Aórtica

\section{ABSTRACT}

Introduction: Transcatheter aortic valve implantation is a less invasive option for aortic valve replacement. The number of transcatheter aortic valve implantations under local anesthesia with sedation has been increasing as the team's experience increases and less invasive accesses are used. The aim of this study is to describe the evolution of the anesthetic technique in patients undergoing transcatheter aortic valve implantation at our center over the years, as which was compared.

Material and Methods: Retrospective study in 149 consecutive patients undergoing transcatheter aortic valve implantation in Hospital Santa Marta (January 2010 to December 2016). Data was collected from the periprocedural records of patients. Patients were stratified according to anesthetic technique.

Results: From our patients' sample, 57.0\% were female, with median age 82 [58 - 95] years. Most patients underwent general anesthesia $(68.5 \%)$. In the local anesthesia with sedation group there was a shorter duration of the procedure (120; [60 - 285] vs $155[30$ - 360]) and a lower number of patients requiring administration of vasopressors $(61.8 \%$ vs $28.3 \%)-p<0.05$. There were no differences regarding length of hospital stay (9 [4 - 59] vs 10 [3 - 87]), periprocedural complications $(66.0 \%$ vs $72.5 \%)$, readmission rate $(4.3 \%$ vs $3.9 \%)$ or 30 -days $(2.1 \%$ vs $4.9 \%)$ and 1 -year mortality $(6.4 \%$ vs $7.8 \%)-p>0.05$. There was an increasing number of transcatheter aortic valve implantations performed under local anesthesia with sedation over the years.

Discussion: The choice of anesthetic technique depends on the patient's characteristics, experience and preference of the team.

Conclusion: Local anesthesia with sedation seems to be associated with similar results as general anesthesia. The increase in the number of transcatheter aortic valve implantations under local anesthesia with sedation seems to follow the trend of lower invasiveness of the procedure.

Keywords: Anesthesia, General; Anesthesia, Local; Heart Valve Prosthesis Implantation; Transcatheter Aortic Valve Replacement

\section{INTRODUÇÃO}

A estenose aórtica grave constitui uma doença valvular cardíaca que afeta aproximadamente $4,4 \%$ da população/ ano com idade superior a 65 anos e que, quando não tratada, se associa a mau prognóstico. ${ }^{1,2}$ Com o envelhecimento da população, estima-se que a prevalência desta patologia venha a aumentar.
A implantação percutânea de válvula aórtica [transcatheter aortic valve implantation (TAVI)] constitui uma opção menos invasiva de substituição valvular. ${ }^{1}$ Está indicada para doentes com estenose aórtica sintomática grave, frágeis e considerados inoperáveis (nomeadamente pela presença de 'aorta de porcelana') bem como para doentes

1. Serviço de Anestesiologia. Hospital de Santa Marta. Centro Hospitalar de Lisboa Central. Lisboa. Portugal.

$\bowtie$ Autor correspondente: Ana Martins. ana.marga.vm@hotmail.com

Recebido: 22 de junho de 2018 - Aceite: 17 de outubro de 2018 | Copyright @ Ordem dos Médicos 2019 
com elevado ou moderado risco cirúrgico. . $^{1,-7}$

Como tal, impõe inúmeros desafios relacionados com o procedimento em si e com a abordagem dos doentes submetidos à implantação valvular.

Os bons resultados obtidos com a colocação endovascular de prótese valvular justificam o crescente número de procedimentos realizados. ${ }^{1}$ Acredita-se que este tratamento venha a ser no futuro uma opção a considerar também em doentes com baixo risco cirúrgico, como resultado da simplificação do procedimento, do desenvolvimento tecnológico e da experiência crescente na execução de TAVIs. ${ }^{1,8}$

A abordagem de substituição de válvula aórtica transcatéter tem-se tornado cada vez menos invasiva (comparativamente à técnica cirúrgica de substituição valvular por via aberta), fazendo sentido que a técnica anestésica acompanhe esta tendência. ${ }^{6,7}$

De um modo geral, durante a fase de implementação destes programas nos diferentes centros europeus, a anestesia geral (AG) tem sido a opção mais consensual, tendo-se assistido a um aumento progressivo do número de TAVIs sob anestesia local com sedação $(A L+S)$ à medida que aumenta a experiência da equipa e se utilizam acessos cada vez menos invasivos. ${ }^{7}$

Existem grandes diferenças entre os vários centros relativamente à intervenção anestésica preferencial, não existindo ainda consenso acerca da melhor opção anestésica. ${ }^{6}$

Ambas as técnicas anestésicas permitem a obtenção de resultados semelhantes no que respeita à taxa de sucesso e de morbimortalidade dos doentes..$^{1,8}$ As diferenças residem nas durações do procedimento e do tempo de internamento hospitalar, em que a utilização de sedação consciente permite uma redução significativa dos mesmos. ${ }^{7,8}$

Este trabalho tem como objetivo a descrição da evolução da técnica anestésica utilizada nos doentes submetidos a TAVI no nosso centro ao longo dos anos, e a comparação dos resultados obtidos entre $a A L+S$ e a $A G$.

\section{MATERIAL E MÉTODOS}

Foi realizado um estudo retrospetivo numa população de 149 doentes consecutivos submetidos a TAVI por via transfemoral ou subclávia no Hospital de Santa Marta entre os meses de janeiro de 2010 e dezembro de 2016, tendo-se considerado como doentes propostos para a realização deste procedimento todos aqueles que apresentavam estenose aórtica grave inoperável ou cujo risco cirúrgico era elevado.

Os dados foram colhidos a partir dos registos intra-procedimento e processo clínico do doente.

$\mathrm{Na}$ análise de dados foram consideradas as características demográficas e ecocardiográficas dos doentes, abordagem anestésica, complicações periprocedimento e resultados a curto e médio prazo.

Os doentes foram monitorizados de acordo com as normas da American Society of Anesthesiologists - ASA. Todos os doentes foram ainda monitorizados com BIS ${ }^{\circledR}$ (bispectral index). Os doentes submetidos a AG tiveram também monitorização com ecocardiografia transesofágica (ETE), sendo que nos submetidos a $A L+S$ a monitorização do procedimento foi feita com recurso a fluoroscopia e ecocardiografia transtorácica (ETT).

Foram colocados cateter venoso central (CVC), cateter arterial radial (CA) e eletrocateter para realização de pacing a todos os doentes.

Os doentes foram submetidos a $A G$ ou $A L+S$. Nos doentes submetidos a $A G$, a indução foi realizada com fentanil $(1-3 \mathrm{mg} / \mathrm{kg})$, propofol $(1-2 \mathrm{mg} / \mathrm{kg})$ e rocurónio $(0,6 \mathrm{mg} /$ $\mathrm{kg}$ ), com intubação orotraqueal. A manutenção foi feita com halogenados ou com propofol em target-controlled infusion (TCl). Os doentes foram extubados no final do procedimento após recuperação anestésica.

Aos doentes submetidos a $\mathrm{AL}+\mathrm{S}$ foram administrados propofol e remifentanil em $\mathrm{TCl}$, com manutenção da ventilação espontânea.

Os maus acessos vasculares foram definidos por tomografia computorizada (TC) pela presença de diâmetros inferiores a $5 \mathrm{~mm}$, e presença de calcificação extensa.

Para a definição das complicações periprocedimento foram utilizadas as definições do Valve Academic Research Consortium-2 (VARC-2). ${ }^{9}$

A mortalidade dos doentes abordados em 2016 foi avaliada até à data da recolha dos dados.

A análise e tratamento estatístico dos resultados foram feitos utilizando o programa informático SPSS 22.0 (IBM Analytics, Armonk, Nova lorque, Estados Unidos da América).

Os doentes foram estratificados de acordo com a técnica anestésica.

São apresentadas as frequências e a média com desvio padrão ou mediana com intervalo interquartil (percentis 25 e 75, IIQ) para as variáveis com distribuição não normal. A normalidade das variáveis quantitativas foi avaliada pelo teste Shapiro-Wilk. As variáveis categóricas são apresentadas em valores absolutos e percentagem. A análise bivariável foi feita pelo teste do qui-quadrado.

As variáveis quantitativas foram comparadas utilizando o teste $t$ ou o teste de Mann-Whitney.

Os resultados foram considerados estatisticamente significativos se $p<0,05$.

\section{RESULTADOS}

A população de estudo incluiu 149 doentes, dos quais 64 pertenciam ao sexo masculino $(43,0 \%)$, tendo mediana de idade de 82 [IIQ 78 - 85] anos. Todos os doentes foram classificados como ASA III $(n=54 ; 36,2 \%)$ ou IV ( $n=95$; $63,8 \%)$.

A totalidade da amostra apresentava estenose aórtica grave como diagnóstico principal, razão pela qual foram submetidos a TAVI. Esta foi realizada por via transfemoral na maioria dos doentes ( $n=145 ; 97,3 \%$ ), tendo os restantes sido abordados por via subclávia por má qualidade dos acessos femorais.

Relativamente ao procedimento, a mediana da sua 
duração foi 150 [IIQ 120 - 180] minutos.

A mediana da duração de internamento na $\mathrm{UCl}$ e do total do internamento foi de 3 [IIQ 2 - 5] e 10 [IIQ 8 - 17] dias, respetivamente.

Nas Tabelas 1, 2 e 3 são apresentadas as características demográficas e ecocardiográficas da população, bem como os dados periprocedimento.
Quanto à técnica anestésica utilizada, foi realizada AG na maioria dos doentes $(n=102 ; 68,5 \%)$. Nos restantes o procedimento decorreu sob AL+S ( $n=47 ; 31,5 \%)$.

Relativamente às caraterísticas clínicas e ecocardiográficas dos doentes, verifica-se que os doentes submetidos a $A L+S$ apresentam menor gravidade segundo a classificação ASA. Ainda neste grupo, é menor a incidência de

Tabela 1 - Características gerais da população (pré-operatórias)

\begin{tabular}{|c|c|c|c|c|}
\hline & & Técnic & tésica & \\
\hline & $\begin{array}{c}\text { Total } \\
(n=149)\end{array}$ & $\begin{array}{c}A G \\
(n=102)\end{array}$ & $\begin{array}{c}\text { AL+S } \\
(n=47)\end{array}$ & $p$ \\
\hline Idade & & & & \\
\hline Mediana [IIQ] & 82 [78 - 85] & 82 [78 - 85] & 81 [77 - 85] & 0,953 \\
\hline Género & & & & 0,37 \\
\hline Masculino & $64(43,0 \%)$ & $41(40,2 \%)$ & $23(48,9 \%)$ & \\
\hline Feminino & $85(57,0 \%)$ & $61(59,8 \%)$ & $24(51,1 \%)$ & \\
\hline Classificação ASA & & & & $0,04^{*}$ \\
\hline ASA 3 & $54(36,2 \%)$ & $31(30,4 \%)$ & $23(48,9 \%)$ & \\
\hline ASA 4 & $95(63,8 \%)$ & $71(69,6 \%)$ & $24(51,1 \%)$ & \\
\hline Classificação EuroScore II & & & & 0,77 \\
\hline Baixo: 0 - 2 & $15(10,1 \%)$ & $9(8,9 \%)$ & $6(12,8 \%)$ & \\
\hline Moderado: 3 - 5 & $49(32,9 \%)$ & $34(33,3 \%)$ & $15(31,9 \%)$ & \\
\hline Alto: $>5$ & $85(57,0 \%)$ & $59(57,8 \%)$ & $26(53,3 \%)$ & \\
\hline HTA & $121(81,2 \%)$ & $78(76,5 \%)$ & $43(91,5 \%)$ & $0,04^{*}$ \\
\hline DM & $43(28,9 \%)$ & $29(28,4 \%)$ & $14(29,8 \%)$ & 0,84 \\
\hline Dislipidemia & $87(58,4 \%)$ & $57(55,9 \%)$ & $30(63,8 \%)$ & 0,38 \\
\hline Obesidade & $28(18,8 \%)$ & $16(15,7 \%)$ & $12(25,5 \%)$ & 0,18 \\
\hline Tabagismo & $12(8,1 \%)$ & $8(7,8 \%)$ & $4(8,5 \%)$ & 1,00 \\
\hline DPOC & $27(18,1 \%)$ & $22(21,6 \%)$ & $5(10,6 \%)$ & 0,17 \\
\hline Critérios de VAD & $33(22,15 \%)$ & $23(22,5 \%)$ & $10(21,3 \%)$ & 1,00 \\
\hline ICC NYHA & & & & 0,20 \\
\hline Classe I-II & $53(35,6 \%)$ & $40(39,2 \%)$ & $13(27,7 \%)$ & \\
\hline Classe III-IV & $96(64,4 \%)$ & $62(60,8 \%)$ & $34(72,3 \%)$ & \\
\hline DRC prévia (TFG $<60 \mathrm{~mL} / \mathrm{min}$ ) & $63(42,0 \%)$ & $38(37,3 \%)$ & $21(47,7 \%)$ & 0,27 \\
\hline DVP & $39(26,2 \%)$ & $32(31,4 \%)$ & $7(14,9 \%)$ & $0,04^{*}$ \\
\hline DAC & $61(40,9 \%)$ & $40(39,2 \%)$ & $21(44,7 \%)$ & 0,59 \\
\hline EAM prévio & $23(15,4 \%)$ & $11(10,8 \%)$ & $12(25,5 \%)$ & $0,03^{*}$ \\
\hline $\mathrm{PCI}$ & $28(18,8 \%)$ & $20(19,6 \%)$ & $8(17,0 \%)$ & 0,82 \\
\hline Cx cardíaca prévia & $14(26,8 \%)$ & $23(22,5 \%)$ & $17(36,2 \%)$ & 0,11 \\
\hline CABG prévio & $31(20,8 \%)$ & $17(16,7 \%)$ & $14(29,8 \%)$ & 0,08 \\
\hline Arritmia & $13(8,7 \%)$ & $10(9,8 \%)$ & $3(6,4 \%)$ & 0,76 \\
\hline FA & $43(28,9 \%)$ & $31(30,4 \%)$ & $12(25,5 \%)$ & 0,70 \\
\hline Alterações neurológicas prévias/AVC/AIT & $21(14,1 \%)$ & $16(15,7 \%)$ & $5(10,6 \%)$ & 0,46 \\
\hline Alterações sensoriais prévias & $6(4,03 \%)$ & $6(5,9 \%)$ & $0(0,0 \%)$ & 0,18 \\
\hline Incapacidade de tolerar DD & $28(18,8 \%)$ & $28(27,5 \%)$ & $0(0,0 \%)$ & $<0,01^{*}$ \\
\hline $\begin{array}{l}\text { Hematócrito pré-operatório } \\
\text { Média }\end{array}$ & $37,03 \pm 5,41$ & $36,65 \pm 5,49$ & $37,84 \pm 5,18$ & 0,22 \\
\hline Maus acessos vasculares definidos por TAC & $39(26,2 \%)$ & $36(35,3 \%)$ & $3(6,4 \%)$ & $<0,01^{*}$ \\
\hline
\end{tabular}

*: $p<0,05$; IIQ: intervalo interquartil [Q25-Q75]; HTA: hipertensão arterial; DM: diabetes mellitus; DPOC: doença pulmonar obstrutiva crónica; VAD: via aérea difícil; ICC NYHA: insuficiência cardíaca congestiva - Classificação New York Heart Association; DRC: doença renal crónica; DVP: doença vascular periférica; DAC: doença arterial coronária; PCI: percutaneous coronary intervention; AVC/AIT: acidente vascular cerebral/acidente isquémico transitório; DD: decúbito dorsal 
Tabela 2 - Dados ecocardiográficos

\begin{tabular}{|c|c|c|c|c|}
\hline & \multirow[b]{2}{*}{$\begin{array}{c}\text { Total } \\
(n=149)\end{array}$} & \multicolumn{2}{|c|}{ Técnica anestésica } & \multirow[b]{2}{*}{$p$} \\
\hline & & $\begin{array}{c}A G \\
(n=102)\end{array}$ & $\begin{array}{c}\text { AL+S } \\
(n=47)\end{array}$ & \\
\hline Fração de ejeção & & & & 0,80 \\
\hline Boa $(\geq 50 \%)$ & $115(78,2 \%)$ & $78(77,2 \%)$ & $37(80,4 \%)$ & \\
\hline Razoável (40 - 49\%) & $19(12,9 \%)$ & $13(12,9 \%)$ & $6(13,0 \%)$ & \\
\hline Má (< 40\%) & $13(8,8 \%)$ & $10(9,9 \%)$ & $3(6,5 \%)$ & \\
\hline Insuficência mitral (moderada/grave) & $59(39,60 \%)$ & $47(46,1 \%)$ & $12(25,5 \%)$ & $0,02^{*}$ \\
\hline $\begin{array}{l}\text { AVA pré-TAVI }\left(\mathbf{c m}^{2}\right) \\
\text { Mediana }[I I Q]^{1}\end{array}$ & $0,63[0,50-0,80]$ & $0,61[0,50-0,80]$ & $0,63[0,50-0,81]$ & 0,87 \\
\hline $\begin{array}{l}\text { PSAP pré-TAVI }(\mathrm{mmHg}) \\
\text { Mediana [IIQ] }{ }^{1}\end{array}$ & $40,00[31,75-51,00]$ & $40,00[30,00-51,00]$ & $40,00[33,50-53,25]$ & 0,29 \\
\hline $\begin{array}{l}\text { Gradiente Ao máx. pré-TAVI (mmHg) } \\
\text { Mediana [IIQ] }{ }^{1}\end{array}$ & $81,00[68,75-97,50]$ & $81,00[69,50-96,50]$ & $83,00[66,00-103,00]$ & 0,72 \\
\hline $\begin{array}{l}\text { Gradiente Ao médio pré-TAVI }(\mathrm{mmHg}) \\
\text { Mediana }[\mathrm{IIQ}]^{1}\end{array}$ & $50,50[42,25-61,75]$ & $51,50[42,25-60,75]$ & $49,50[41,50-63,50]$ & 0,89 \\
\hline $\begin{array}{l}\text { AVA pós-TAVI }\left(\mathrm{cm}^{2}\right) \\
\text { Mediana }[\mathrm{IIQ}]^{1}\end{array}$ & $1,30[1,10-1,90]$ & $1,20[1,00-1,45]$ & $1,60[1,20-2,00]$ & 0,09 \\
\hline $\begin{array}{l}\text { PSAP pós-TAVI }(\mathrm{mmHg}) \\
\text { Mediana [IIQ] }^{1}\end{array}$ & $38,00[31,00-45,00]$ & $38,00[31,00-44,00]$ & $40,00[31,75-47,00]$ & 0,41 \\
\hline $\begin{array}{l}\text { Gradiente Ao máx. pós-TAVI (mmHg) } \\
\text { Mediana [IIQ] }]^{1}\end{array}$ & $17,50[13,25-24,75]$ & $19,50[14,00-26,00]$ & $15,00[13,00-19,75]$ & 0,06 \\
\hline $\begin{array}{l}\text { Gradiente Ao médio pós-TAVI (mmHg) } \\
\text { Mediana [IIQ }]^{1}\end{array}$ & $10,00[7,00-13,00]$ & $10,00[8,00-14,50]$ & $8,50[7,00-12,25]$ & 0,23 \\
\hline
\end{tabular}

*: $p<0,05$; AVA: área valvular aórtica; PSAP: pressão sistólica artéria pulmonar; Gradiente Ao: gradiente aórtico;

hipertensão arterial (HTA), doença vascular periférica (DVP), incapacidade de tolerar decúbito dorsal e de maus acessos vasculares. No entanto, estes apresentam mais história prévia de enfarte agudo do miocárdio (EAM).

$\mathrm{Na}$ comparação das duas técnicas anestésicas verificaram-se diferenças na duração do procedimento $(p<0,05)$, tendo este menor duração quando aplicada $A L+S$, e em relação à necessidade de vasopressores $(p<0,05)$ - Tabela 3. O número de doentes a requerer suporte com fármacos vasopressores foi maior no grupo dos submetidos a AG. Não se verificaram diferenças entre a $A G$ e a $A L+S$ para outras variáveis, nomeadamende idade, género, EuroScore II, abordagem TAVI, duração do internamento na $\mathrm{UCI}$ e total, tipos de complicações periprocedimento, taxa de reinternamento, e mortalidade aos 30 dias e um ano $(p>0,05)$.

Da análise das técnicas anestésicas utilizadas durante o tempo no qual decorreu este estudo, verificou-se um aumento progressivo do número de procedimentos realizados sob $A L+S$ relativamente à $A G$ (em 2010 todos os procedimentos foram realizados com AG, enquanto que em 2015 $50,0 \%$ dos procedimentos foram realizados sob $A L+S)-$ Fig. 1.

De entre as complicações mais frequentes, destacam-se as perdas sanguíneas intra-procedimento (9,3\%), a lesão renal aguda - LRA $(3,1 \%)$, arritmias como a fibrilhação auricular (8,6\%), complicações vasculares (nomeadamente ao nível do membro inferior na abordagem transfemoral $11,0 \%)$ e eventos trombo-embólicos $(3,8 \%)$, todas elas determinantes para o resultado pós-operatório de cada doente.
No grupo de doentes submetidos a AG as complicações mais frequentes foram, por ordem decrescente, a ocorrência de arritmias $(39,2 \%)$ e hemorragia major $(34,3 \%)$, complicações vasculares $(24,5 \%)$ e a necessidade de colocação de PMD (22,5\%).

Já no grupo de doentes submetidos a $A L+S$ as complicações mais frequentes incluiram a ocorrência de hemorragia major (31,9\%), arritmias $(27,7 \%)$ e complicações vasculares $(23,4 \%)$.

Destaque para a necessidade de conversão de $A L+S$ para AG em três doentes: 1 por reação alérgica à cefazolina e 2 por complicações vasculares com repercussão hemodinâmica.

Não se verificaram complicações inerentes à técnica anestésica em nenhum doente.

\section{DISCUSSÃO}

Para os doentes propostos para TAVI, a escolha da técnica anestésica varia consoante o centro, caraterísticas do doente e a preferência da equipa.

Não existindo ainda consenso no que diz respeito à melhor técnica anestésica a realizar para esta intervenção, cabe ao anestesiologista a seleção daquela que considera mais adequada e com a qual toda a equipa se sente mais confortável, sempre tendo em atenção as comorbilidades, características pré-operatórias e avaliação ecocardiográfica do doente, tendo como objetivo máximo a sua segurança e conforto.

Este trabalho demonstrou o crescente número de TAVIs realizadas sob $A L+S$ no nosso centro. 
Tabela 3 - Dados periprocedimento

\begin{tabular}{|c|c|c|c|c|}
\hline & \multirow[b]{2}{*}{$\begin{array}{c}\text { Total } \\
(n=149)\end{array}$} & \multicolumn{2}{|c|}{ Técnica anestésica } & \multirow[b]{2}{*}{$p$} \\
\hline & & $\begin{array}{c}A G \\
(n=102)\end{array}$ & $\begin{array}{c}\text { AL+S } \\
(n=47)\end{array}$ & \\
\hline Abordagem TAVI & & & & 0,31 \\
\hline Transfemoral & $145(97,3 \%)$ & $98(96,1 \%)$ & $47(100 \%)$ & \\
\hline Subclávia & $4(2,7 \%)$ & $4(3,9 \%)$ & $0(0,0 \%)$ & \\
\hline $\begin{array}{l}\text { Duração do procedimento (minutos) } \\
\text { Mediana [IIQ] }{ }^{1}\end{array}$ & $150,00[120,00-180,00]$ & $155,00[135,00-195,00]$ & $120,00[105,00-150,00]$ & $<0,01^{*}$ \\
\hline $\begin{array}{l}\text { Duração internamento UCI (dias) } \\
\text { Mediana [IIQ] }{ }^{1}\end{array}$ & $3,00[2,00-5,00]$ & $3,00[2,00-5,00]$ & $3,00[2,00-4,00]$ & 0,26 \\
\hline $\begin{array}{l}\text { Duração do internamento (dias) } \\
\text { Mediana [IIQ] }{ }^{1}\end{array}$ & $10,00[8,00-17,00]$ & $10,00[8,00-18,50]$ & $9,00[7,00-14,00]$ & 0,49 \\
\hline Necessidade de vasopressores & $76(51,4 \%)$ & $63(61,8 \%)$ & $13(28,3 \%)$ & $<0,01^{*}$ \\
\hline Complicações periprocedimento* & $105(70,5 \%)$ & $74(72,5 \%)$ & $31(66,0 \%)$ & 0,44 \\
\hline Hemorragia major & $50(33,56 \%)$ & $35(34,3 \%)$ & $15(31,9 \%)$ & 0,85 \\
\hline Arritmias & $53(35,57 \%)$ & $40(39,2 \%)$ & $13(27,7 \%)$ & 0,20 \\
\hline Colocação PMD & $30(20,13 \%)$ & $23(22,5 \%)$ & $7(14,9 \%)$ & 0,38 \\
\hline Complicações vasculares & $36(24,2 \%)$ & $25(24,5 \%)$ & $11(23,4 \%)$ & 1,00 \\
\hline Complicações infeciosas & $28(18,8 \%)$ & $21(20,6 \%)$ & $7(14,9 \%)$ & 0,50 \\
\hline LRA - Classificação AKIN & $22(14,77 \%)$ & $17(16,7 \%)$ & $5(10,6 \%)$ & 0,10 \\
\hline 1 & $12(8,1 \%)$ & $11(10,8 \%)$ & $1(2,1 \%)$ & \\
\hline 2 & $5(3,36 \%)$ & $3(2,9 \%)$ & $2(4,3 \%)$ & \\
\hline 3 & $5(3,36 \%)$ & $3(2,9 \%)$ & $2(4,3 \%)$ & \\
\hline Insuficiência respiratória & $4(2,7 \%)$ & $4(3,9 \%)$ & $0(0,0 \%)$ & 0,31 \\
\hline AVC/AIT & $3(2,0 \%)$ & $2(2,0 \%)$ & $1(2,1 \%)$ & 1,00 \\
\hline SCA & $7(4,7 \%)$ & $5(4,9 \%)$ & $2(4,3 \%)$ & 1,00 \\
\hline Complicações - Outras & $16(10,7 \%)$ & $9(8,8 \%)$ & $7(14,9 \%)$ & 0,27 \\
\hline PCR & $5(3,4 \%)$ & $4(3,9 \%)$ & $1(2,1 \%)$ & 1,00 \\
\hline Transfusão & $46(30,9 \%)$ & $32(31,4 \%)$ & $14(29,8 \%)$ & 1,00 \\
\hline Reinternamento & $6(4,0 \%)$ & $4(3,9 \%)$ & $2(4,3 \%)$ & 1,00 \\
\hline Morte aos 30 dias & $6(4,0 \%)$ & $5(4,9 \%)$ & $1(2,1 \%)$ & 0,67 \\
\hline Morte a 1 ano & $11(7,4 \%)$ & $8(7,8 \%)$ & $3(6,4 \%)$ & 1,00 \\
\hline
\end{tabular}
UCl: unidade cuidados intensivos; PMD: pacemaker definitivo; LRA: lesão renal aguda; SCA: síndrome coronária aguda; PCR: paragem cardiorrespiratória.
$*: p<0,05$

Verificou-se que o grupo de doentes submetidos a $A L+S$ apresentou menores tempos de procedimento e menor necessidade de administração de vasopressores.

A diferença verificada na duração do procedimento assume-se como sendo devida a um maior consumo de tempo associado à indução e recuperação anestésicas nos doentes submetidos a AG.

Já a menor necessidade de fármacos vasopressores no grupo de doentes de $A L+S$ deve-se, possivelmente à vantagem da sedação em limitar os efeitos cardiodepressores e vasodilatadores associados aos anestésicos em doentes com estenose aórtica grave.

De acordo com outros estudos, a AL+S apresenta ainda outros benefícios tais como menor duração do internamento, bem como de complicações peri-procedimento e taxa de mortalidade a curto e longo prazo, que no entanto não se verificaram neste grupo de doentes. ${ }^{7}$

$A A L+S$ acresce ainda vantagens no período pós- -procedimento ao promover uma recuperação mais rápida do doente e, consequentemente, uma deambulação mais precoce, o que contribui para a diminuição dos tempos de internamento e custos associados. ${ }^{8}$

Outro fator que está ligado ao aumento do número de procedimentos realizados sob $A L+S$ é o aumento da experiência da equipa de cardiologia e anestésica na realização de TAVIs; isto faz prever que, no futuro, um número cada vez maior de procedimentos venha a ser realizado com técnicas anestésicas cada vez menos invasivas. Dados de estudos prévios sugerem a preferência da realização de AG durante a curva de aprendizagem dos operadores por ajudarem a manter o doente imóvel, permitirem o controlo dos movimentos respiratórios e a monitorização com ETE. 8,11

$\mathrm{Na}$ seleção da técnica anestésica importa ainda ter sempre presente o tipo de abordagem, sendo a femoral a mais comum.

À semelhança do que se passa no Hospital de Santa 


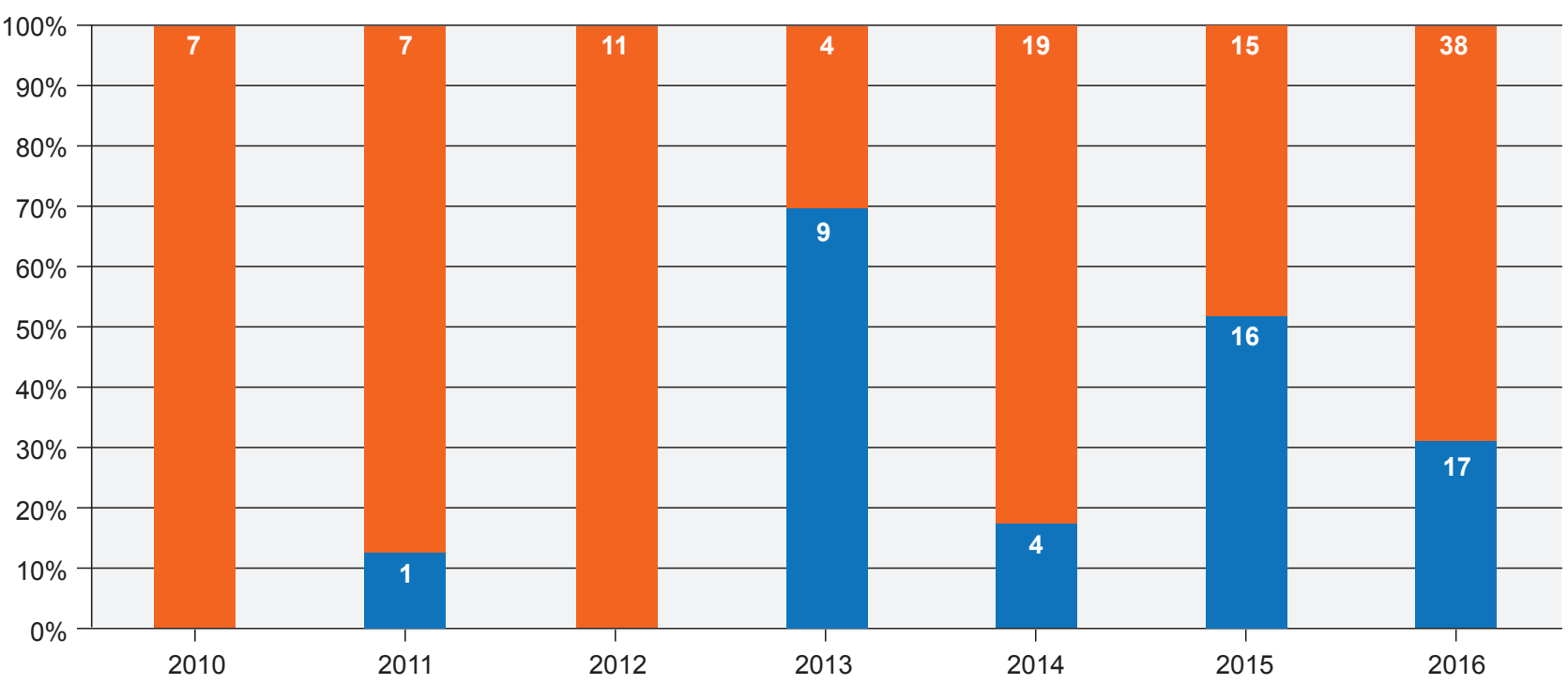

$A L+S \quad A G$

Figura 1 - Número de TAVIs realizados por ano e técnica anestésica

Marta, Ruggeri et al defendem a utilização de AG em condições específicas determinadas pela avaliação clínica do anestesiologista e operador, estando a ETE reservada para casos de elevado risco ou para situações com maior potencial para o surgimento de complicações. ${ }^{11}$

De referir que todos os autores que defendem a utilização de AL concordam com a necessidade de o anestesiologista ter que estar preparado para a indução de AG a qualquer momento, caso a situação o exija. ${ }^{11}$

A baixa taxa de necessidade de conversão para AG constitui outro dos aspetos a favor desta técnica anestésica na realização de TAVIs, sendo que nenhum dos casos de conversão ocorreu por causas anestésicas.

Importa realçar os bons resultados obtidos com a TAVI no nosso centro comparativaente à substituição valvular aberta (SVA) no que diz respeito a complicações como perdas sanguíneas intra-procedimento $(9,3 \%$ vs $19,5 \%$ na SVA), LRA ( $3,1 \%$ vs $3,2 \%$ na SVA) e arritmias como a fibriIhação auricular (8,6\% vs $16,0 \%$ na SVA). A TAVI evidenciou maior número de complicações vasculares (nomeadamente ao nível do membro inferior na abordagem transfemoral - $11,0 \%$ vs $3,2 \%$ na SVA) e eventos tromboembólicos (3,8\% vs $2,1 \%$ na SVA). 6,10

Como limitações deste estudo realça-se o seu caráter retrospetivo e o facto destes resultados refletirem a experiência e os resultados das TAVIs realizadas num único centro. Considerando que a experiência dos cardiologistas é fundamental na escolha da técnica anestésica, torna-se fundamental a existência de estudos multicêntricos em centros com diferente volume de TAVIs realizadas de forma a avaliar a aplicabilidade das diferentes opções anestésicas.

Além do mais, a amostra é constituída por um reduzido número de doentes, sendo importante a existência de uma população maior e de acompanhamento por período de tempo mais longo de forma a determinar o impacto da técnica anestésica no resultado a longo prazo.

Considerando ainda que a $\mathrm{AL}+\mathrm{S}$ inclui um largo espetro de intervenções, é igualmente necessária a especificação dos diferentes fármacos utilizados neste âmbito de forma a avaliar e estudar as interferências de cada um nos resultados obtidos.

Seria ainda importante avaliar o pós-operatório imediato no grupo de doentes submetidos a $A G$ e $A L+S$ de forma a verificar se existem diferenças nomeadamente no que diz respeito a graus de sedação e estados confusionais. ${ }^{12}$

Todos estes aspetos têm relevo na atualidade, na medida em que os fármacos de curta duração de ação utilizados para AG têm propriedades farmacocinéticas que permitem indução e recuperação anestésica cada vez mais céleres associadas a um perfil de segurança cada vez maior, proporcionando uma extubação imediata pós-procedimento. ${ }^{12}$

\section{CONCLUSÃO}

A realização de TAVIs tem aumentado progressivamente, sendo cada vez mais selecionada nos doentes com estenose aórtica grave.

Os resultados obtidos evidenciam vantagens no grupo de doentes submetidos a $A L+S$ na diminuição da duração do procedimento e da necessidade de administração de fármacos vasopressores.

A escolha da técnica anestésica não parece interferir com o resultado da TAVI.

Com o ganho crescente de experiência na abordagem dos doentes submetidos a TAVI's por parte dos cardiologistas e anestesiologistas, e perante as vantagens apresentadas, a seleção de $\mathrm{AL}+\mathrm{S}$ como técnica anestésica menos invasiva nos doentes submetidos a TAVI tem vindo a aumentar no nosso centro.

Tornam-se necessários estudos multicêntricos que, 
tendo presente o grau de experiência de cada centro, permitam determinar as características pré-operatórias determinantes para a seleção da técnica anestésica bem como a avaliação dos resultados das TAVI's a médio e longo prazo de forma a determinar qual a técnica anestésica mais adequada.

É fundamental que os anestesiologistas tenham um papel participativo no cuidado deste grupo de doentes de elevado risco, em todo o período peri-procedimento, desde a avaliação anestésica - incluindo a avaliação de fragilidade - até ao seguimento e avaliação do impacto das intervenções especificamente anestésicas. É igualmente importante que as técnicas anestésicas acompanhem a tendência observada nos procedimentos endovasculares de substituição valvular, no sentido de invasibilidade progressivamente menor.

\section{PROTECÇÃO DE PESSOAS E ANIMAIS}

Os autores declaram que os procedimentos seguidos estavam de acordo com os regulamentos estabelecidos pelos responsáveis da Comissão de Investigação Clínica e Ética e de acordo com a Declaração de Helsínquia da Associação Médica Mundial.

\section{CONFIDENCIALIDADE DOS DADOS}

Os autores declaram ter seguido os protocolos do seu centro de trabalho acerca da publicação de dados. Consentimento do doente obtido.

\section{CONFLITOS DE INTERESSE}

Os autores declaram não terem qualquer conflito de interesse relativamente ao presente artigo.

\section{FONTES DE FINANCIAMENTO}

Os autores negam bolsas ou subsídios que contribuíram para a realização do trabalho.

et al. Conscious sedation versus general anesthesia for transcatheter aortic valve replacement. Circulation. 2017;136:2132-40.

\section{REFERÊNCIAS}

1. Luscher T. TAVI: from an experimental procedure to a standard of care. Eur Heart J. 2018;39:2605-8.

2. Peeters F, Meex S, Dweck M, Aikawa E, Crijns H, Schurgers LJ, et al. Calcific aortic valve stenosis: hard disease in the heart. Eur Heart J. 2018;39:2618-24.

3. Baumgartner H, Falk V, Bax J, Bonis M, Hamm C, Holm P, et al. 2017 ESC/EACTS Guidelines for the management of valvular heart disease The Task Force for the Management of Valvular Heart Disease of the European Society of Cardiology (ESC) and the European Association for Cardio-Thoracic Surgery (EACTS). Eur Heart J. 2017;38:2739-91.

4. Aksoy M, Ince I, Ahiskalioglu A, Dogan N, Colak A, Sevimli S. Transcatheter aortic valve implantation: first applications and short term outcomes in our clinic. Eurasian J Med. 2015;47:91-8.

5. Mack MJ, Brennan JM, Brinfdis R, Carroll J, Grover F, Shahian D, et al. Outcomes following transcatheter aortic valve replacement in the United States. JAMA. 2013;310:2069-77.

6. Dall'Ara G, Eltchaninoff H, Moat N, Laroche C, Goicolea J, Ussia GP, et al. Local and general anaesthesia do not influence outcome of transfemoral aortic valve implantation. Int J Cardiol. 2014;177:448-54.

7. Hyman M, Vemulapalli S, Szeto W, Stebbins A, Patel P, Matsouaka R,
8. Frohlich GM, Lansky AJ, Webb J, Roffi M, Toggeiler S, Reinthaler M, et al. Local versus general anesthesia for transcatheter aortic valve implantation (TAVR) - systematic review and meta-analysis. BMC Med. $2014 ; 12$.

9. Kappetein AP, Head SJ, Généreux P, Piazza N, Mieghem NM, Blackstone $\mathrm{EH}$, et al. Updated standardized endpoint definitions for transcatheter aortic valve implantation: the Valve Academic Research Consortium-2 consensus document. J Thorac Cardiovasc Surg. 2013;145:6-23.

10. Smith C, Leon M, Mack M, Miller D, Moses J, Svensson L, et al. Transcatheter versus surgical aortic-valve replacement in high-risk patients. N Engl J Med. 2011;364:2187-98.

11. Ruggeri L, Gerli C, Franco A, Barile L, Magnano di San Lio MS, Villari $\mathrm{N}$, et al. Anesthetic management for percutaneous aortic valve implantation: an overview of worldwide experiences. HSR Proc Intensive Care Cardiovasc Anesth. 2012;4:40-6.

12. Buffon KA, Augoustides JG, Cobey FC. Anesthesia for transfemoral aortic valve replacement in North America and Europe. J Cardiothorac Vasc Anesth. 2013;27:46-9. 